\title{
On Replacing Peer Review with Legal Challenge in Scientific Research: An Opinion
}

\author{
Phillp J. Held, Friedrich K Port, Robert A. Wolfe, Nathan W. Levin, and Marc N Turenne \\ Universlty of Michigan Schoois of Medicine and Public Health, Ann Arbor, Michigan (PJH, FKP, RAW, MNT) and Beth \\ isroel Medical Center, New York New York (NML)
}

We appreciate the invitation from the editor of Seminars in Dialysis to express our opinions about a topic that is very acute in our personal and professional lives. In March 1993, Minntech Corporation, which sells Renalin ${ }^{\infty}$, filed a lawsuit in the United States District Court for the District of Maryland against the five authors of this editorial (plus one other person). Minntech is seeking over one million dollars in damages from us as individuals based on our reports of scientific research findings. The suit claims damages to Minntech because our research had suggested an association of mortality risks with the use of certain reuse agents, including Renalin ${ }^{\star}$, in conventional dialyzers in freestanding dialysis units. Minntech subsequently publicized many of its allegations in letters to various journals $(1-4)$.

Until now, in our pursuit of scientific objectivity, we have abstained from publishing any written response to what we view as an unfounded legal suit. Our primary goal to date has been to publish the scientific results through the peer review process and to let the renal community judge the merit of this research. In fact, at the time of this writing the scientific research paper in question has been accepted and is in press at the American Journal of Kidney Diseases (5).

Minntech's decision to pursue litigation has been a subject of comment in this and other journals. A recent editorial by $\mathrm{C}$. $\mathbf{M}$. Kjellstrand in a previous issue of this journal (6) describes some of Minntech's allegations. Other publications have applauded the right for a legal challenge to scientific research (7) or have provided possible mechanisms and discussion explaining the associations that we reported $(8-10)$. These publications follow other related research (11-15).

Although we are in agreement with much of Dr. Kjellstrand's editorial, there are two assumptions in Dr. Kjellstrand's editorial that require clarification and correction. Furthermore, we believe that the largely unilateral publicity and the assertions by Minntech should not go unanswered.

\section{Dr. Kellstrand Suggests that the Research May Have Been Presented Too Hastily}

Quite the contrary is the case. At the time of our discussions with representatives of several Federal

Address correspondence to: Phillp J. Held PhD. University of Michigan, 315 West Huron-suite 240. Ann Arbor, Ml 48103.

Seminars in Dlahssis-Vol 7. No 2 (Mar-Apr) 1994 pp 98-102 agencies, the research process regarding reuse germicides and patient outcomes had been underway for more than a year. The results of a pilot study had been reviewed by the Scientific Advisory Committee of the USRDS in June and September of 1992. The national study that was the focus of discussions and review with federal health agencies (our present study) followed up this earlier research, and analytical work had been intensely pursued for at least five months.

After discussing and reviewing our findings with federal health officials, we were directed by the Health Care Financing Administration (HCFA, the funding agency) to meet with representatives from HCFA, the Centers for Disease Control and Prevention (CDC) and the Food and Drug Administration (FDA). To further discuss these results, we presented these findings to epidemiologists, statisticians, and other representatives of these agencies at several meetings in September 1992. Throughout this process, we were instructed by the sponsors of the research (HCFA) that the results were to be considered confidential. After these meetings, the FDA concluded that it was appropriate to schedule an invited session on October 8, 1992. At the FDA's request, the authors presented their research findings at that meeting. The findings were also presented to the dialysis community at the Annual Meeting of the American Society of Nephrology (ASN) in November 1992. In short, the study was thorough and precise, with careful and deliberate attention to detail.

The authors communicated with Minntech representatives on numerous occasions in October and November 1992 and also met with several Minntech executives. During this period, the authors explained their analytical methods and results to Minntech, provided statistical output to Minntech, and considered Minntech's disagreements with the research. As part of a prepublication review process, a draft version of the study manuscript was sent in February 1993 for comment to federal officials who had been involved in the previous review process. A copy was also sent to Minntech and, as is normal in the scientific process, copies were also sent to a few knowledgeable researchers in the renal community for review. Minntech did not provide comments on the draft and instead proceeded with a lawsuit.

In the course of studying and reporting data with potentially significant public health implications, we have proceeded in a careful, responsible, and 
ethical manner. Suppressing, delaying, or otherwise withholding these findings from federal agencies or the dialysis community would have been wholly inconsistent with our obligations as researchers and with the interests of patients and dialysis providers. In our opinion, stopping or delaying publication of this paper and chilling open discussion of these findings have been major objectives of Minntech's lawsuit against the six authors.

\section{Dr. Kjellstrand's Editorial Addressed Minntech's Asserted Inability to Gain Access to the Underlying Data Used in our Analyses}

We agree with the principle that data upon which public health findings are based should be available for evaluation and assessment by the medical community. At the same time, because much of these data involves medical information about identified patients, there is an obvious need for appropriate safeguards to protect the privacy of these patients. We have at all times willingly cooperated with both Minntech and federal health agencies in reconciling these interests.

All researchers who work with patient data of the kind used in this research must, according to federal law (the Privacy Act, 5 U.S.C. Section 552 a [i] [3], sign a confidentiality agreement not to release the data. The authors were therefore bound by federal law to keep the data confidential and not release them (Minntech was informed of this before it filed its lawsuit). Since the authors support the right of all researchers to carry out their own analysis of these data files, the authors met with representatives from Minntech and the Federal government in November 1992 to explain exactly how to go about gaining access to the data through submission of a research protocol.

At Minntech's request, the authors referred Minntech to a research firm that had had prior access to the federal data and could assist Minntech in obtaining and evaluating the data using the research protocol route that is open to all researchers. Minntech engaged this firm and notified its clients, the government, and the renal community that this firm, using the federal data, would be conducting its own analysis of the questions raised by our paper. Shortly thereafter, however, Minntech decided not to have this firm proceed with its plan to submit a research protocol, obtain the data, and conduct statistical research. We do not know the basis for Minntech's decision. We have seen no evidence that Minntech notified its clients or the renal community of its change in plans.

Minntech instead chose to pursue litigation against the United States (in addition to its separate litigation against the authors), seeking the underlying medical data under the Freedom of Information Act. For present purposes, we need not speculate on the merits of that case or on Minntech's motives for pursuing litigation rather than following the more conventional research process for obtaining access to these data. It is clear, however, that we have in no way concealed the data used in our analyses from Minntech, and any suggestion to that effect is untrue. To the contrary, we made substantial efforts to help Minntech understand our research, gain access to the underlying data, and engage in independent analysis.

\section{Minntech Has Incorrectly Implied That There Was a Conflict of Interest and the Research Was Blased Against Minntech}

Minntech has also attempted to discredit the research findings by attacking the messengers. Minntech's implication that any of the six researchers was biased or had a conflict of interest is not supported by the facts. (Notably, while Minntech was asserting this conflict of interest, Minntech itself repeatedly relied on research findings and presentations by Alan Collins, MD, often without any mention of the fact that Dr. Collins had been a paid consultant for Minntech.)

The study initially presented at the FDA and ASN meetings was for patients treated in freestanding units which used predominantly conventional dialyzers. This subpopulation of the total U.S. dialysis population was chosen before any analyses were done for two reasons: (i) since the database had limited information on comorbidities (age, race, gender, cause of renal failure), patients in freestanding units were chosen as a more stable and more homogeneous population than if patients treated in hospital units had been included; and (ii) since highflux dialyzers are rarely used without reprocessing, we considered it difficult to find an adequate control group of units employing only single use. Thus our design was rational, consistent with previous research designs, and not driven by bias or selection of the most striking results. Subsequent analyses of other patient groups, including patients in hospitalbased dialysis units and patients in units using highflux dialyzers, are also reported in the final publication (5).

Regarding the authors and alleged conflicts of interest stemming from financial and other economic forces related to the issue of dialyzer reuse and Minntech, let us clearly state the facts.

First, from the outset of this research, the authors never had a predisposition against dialyzer reuse. To the contrary, several of the authors had previously published findings supportive of reuse (1617). (Notably, Minntech has cited some of these pro-reuse findings in its product literature; apparently these favorable findings were not considered the product of bias). Indeed, after our research was presented to the government and the ASN, and prior to the filing of the lawsuit, Dr. Levin wrote an editorial in this journal that was supportive of reuse and urged that our findings be interpreted cautiously (18). Dr. Levin's editorial cautioned (as we have in all presentations of these research findings) that the associations found in our analysis should not be interpreted as showing a causal relationship between Renalin ${ }^{\oplus}$ reuse and mortality. 
Second, our dialysis reuse research has been funded exclusively by agencies of the United States Government. No corporation or other private organization had any involvement in the research, with the exception of our request for comments from Minntech and another company that currently sells a glutaraldehyde-based germicide. ${ }^{1}$

Finally, as to other activities of the authors, Drs. Held, Port, and Levin have spoken at professional meetings sponsored by manufacturers of dialysis products for which they received a speaker's honorarium and travel expenses. In the case of Drs. Held and Port, the sponsor was the Baxter Health Care Corporation; for Dr. Levin the sponsor was Fresenius USA, Inc. Baxter has provided research support to the University of Michigan, Dr. Port's professional base; Fresenius AG, a German corporation, has provided research support to Beth Israel Medical Center, the professional base of Dr. Levin. Neither this research support nor the speaking engagements had anything to do with dialyzer reuse activities. In addition, Dr. Wolfe has consulted with National Medical Care, Inc., on issues unrelated to dialyzer reuse. Beth Israel Medical Center has been involved in the development of dialyzer heat sterilization, a method for the reprocessing of dialyzers. This research has been conducted at no financial advantage to Dr. Levin. No research support has been paid to Dr. Levin or to Beth Israel Medical Center for dialyzer reprocessing research. Dr. Levin has not received consultant fees or any direct or indirect payment from Fresenius AG or Fresenius USA, Inc. Dr. Levin owns less than $1 / 10$ of $1 \%$ of the outstanding common stock of Fresenius USA, Inc., the shares of which are publicly traded. None of the authors have ever owned Minntech stock or had options to buy or sell Minntech stock.

In reporting these facts, we go far beyond the disclosure that is required in even the most demanding research journals. (As noted above, we also go far beyond Minntech's treatment of its direct financial relationship with various physicians.) We do so because it is essential that our scientific findings be evaluated on their merits-not on the basis of unfounded rumor or personal attacks on the authors.

The reality is that we were motivated to carry out and report this research solely by our interest in scientific research and in arriving at the correct science regarding the relative effectiveness of different end-stage renal disease therapies. Even a strong bias could not have modified the epidemiologic and statistical analysis of over 60,000 patient years of experience or the results of the study. The conclusions we reached from these data reflect our best professional judgment, and our analysis and conclusions have been extensively reviewed by profes-

\footnotetext{
${ }^{1}$ The currently ovallable glutaradehyde-bosed germicide wos not marketed at the time of our study, and one of the glutaraldehydebased germicides that was marketed at the time of our study had been removed from the market by the FDA prior to our report.
}

sional peers in the renal community as well as professional and governmental organizations.

In presenting these findings in oral presentations and a forthcoming paper, we have been candid and forthright about the limitations of epidemiological studies, including this one. We repeatedly made considerable effort to emphasize that no causal link between use of a germicide and mortality risk could be inferred from these studies. We emphasized from the beginning that the variation in outcomes from unit to unit was large and that there were some dialysis units using all germicides, including Renalin that had low mortality compared with the average of units not reusing.

In short, however much Minntech may dislike or disagree with our conclusions, we find its public effort to discredit these findings by attacking the authors to be without justification. It is irresponsible and totally unfounded for Minntech to assert in a lawsuit and in letters to journals-as it has-that these researchers knowingly produced and disseminated false research.

\section{Germicide Efficacy Versus Effectiveness}

One of the points we have made repeatedly is that our associations cannot distinguish between the effects of the germicide used in reprocessing and the manner in which the germicide is used. The latter might be called the "user factor." Our research focused on the "effectiveness" of reuse, which includes both the "efficacy" of the product and the manner in which the product is used (user factor) in nonselected U.S. centers.

Minntech has frequently made the claim that, if used as directed, Renalin is safe. Implicit in this claim is that Minntech is talking about "efficacy," in contrast to our focus on "effectiveness," which includes "efficacy" as well as the "user factor." Indeed, we have frequently pointed out that our results may not have been caused by the germicide itself, but instead may be explained by the manner in which it is used. Even if Minntech is correct about the efficacy of Renalin', the statistical associations we have reported would still point to substantial public health problems that may be present because of misuse of the product. Clearly the FDA policy response suspected a user problem since it focused on the methods of proper use of Renalin*.

One of the major reasons for presenting and publishing the results of studies such as ours is to allow the scientific community to examine the evidence and consider what mechanisms might be responsible for the observed association. To bring up an analogy, if use of an efficacious drug were associated with an increase in mortality in a substantial subgroup of patients it would certainly call for intense scrutiny. Failure to report such a finding would be considered unethical. It was in this spirit that these results were discussed and reviewed by the authors with the HCFA, the CDC, the FDA, and the renal community. Should such reports be suppressed for fear of lawsuits? In this regard, Dr. 
Kjellstrand's editorial makes a very good point that the current litigation will only slow down the process of finding the truth in this matter.

\section{What Are the Lessons of this Experience for Research?}

We did not invite or welcome the litigation from Minntech. At the direction of federal sponsors and a scientific advisory committee, we diligently engaged in research addressing a significant public health issue and reported the findings accurately and responsibly to federal health agencies and the dialysis community.

Minntech's decision to resort to litigation in which it seeks more than one million dollars in alleged damages from the investigators has diverted too much energy and money from research and has inserted fear of litigation into the scientific process. The defendants' legal costs have been very substantial over the last year, even before entering the deposition phase. Trial is not likely until late 1994. One can only speculate what the ultimate expense of this litigation will be. We have been fortunate to have support from our institutions, which have not flinched at this challenge to the research process. What would have happened if we had not been so fortunate? What would happen to other researchers not as well supported by their institutions? And more importantly, what does the precedent of this lawsuit mean for the research process? At least one other research group has federal support to examine dialyzer reuse. Try to imagine the climate they face as they prepare to report their findings. Dr. Kjellstrand's editorial provided a good summary of what this lawsuit means for the research process.

There are at least two qualities of the legal system that are very detrimental to the research process. First, resolving legal disputes is costly and extremely time-consuming; second, the legal system is not an efficient machine to find truth in complex scientific matters.

The fact that our institutions have agreed to pay for legal fees so far does not make this litigation "free" to society. Just as the cost of medical malpractice is passed on in higher prices to consumers and taxpayers, the cost of litigation in research will be passed on to others. This country does not need to have a substantial new cost burden in our current health care system. Just when our medical system is focusing on more studies of outcomes research such as our own project for the goal of a more efficient health care system, a new wrench is thrown into the works. Scientific issues will henceforth be threatened by the courts!

What if errors are made in scientific research? As Dr. Kjellstrand noted, this will sometimes occur, but scientific processes exist to correct such errors. We suspect that this process is robust enough to deal with errors of many types, even those that could arise from conflicts of interest that are present in industry-sponsored research.
One process is the manner in which studies are repeated and reproduced as part of scientific inquiry. We believe that Minntech has the right to the federal data under the same conditions with which we and all researchers have had to comply. Minntech should be able to confirm or challenge our results. There are established processes for researchers who want to gain access to the data, processes that assure appropriate confidentiality. Instead of relying on a research proposal, however, Minntech has resorted to much more heavy-handed attacks, which pose different threats to society. Scientists differ with each other often. To challenge published research, a scientist typically builds another experiment with other data, other methods, and/or other theoretical models. Over time, the errors of the past are bypassed, refuted, and replaced by more complete and accurate results.

Our advice to Minntech before the litigation was to take the high road. This approach might have included making a public statement saying it thought these six researchers were wrong and that Minntech was going to show them to be wrong. We further advised them that if their investigation found that there is something to these associations and that the problem lies with Minntech products or how they are used, Minntech as a reputable and respected company should have fixed matters. That advice still stands, but we now believe that Minntech should also repair some of the damage it has done to the scientific research process by reimbursing the Urban Institute, the University of Michigan, and Beth Israel Hospital for legal expenses. The current message Minntech has given to the research community is that those who report adverse facts about experience with Minntech products may suffer legal expenses from litigation and unfounded accusations in the literature. Failure to reverse these actions and reimburse the expenses already incurred would stand as a signal to those who might naïvely believe that they could comment on their experience with Renalin ${ }^{\star}$ in a free exchange of facts.

Our scientific advice to Minntech at the initial presentations was to test whether the reuse procedures with Renalin differed between units with high and low standardized mortality rates. We proposed that we identify dialysis units using Renalin with the highest and lowest standardized mortality rates and blind Minntech to their grouping. On-site data collection and analysis had a very high probability of determining whether the "user factors" may be an explanation for the observed differences in mortality. It was our belief that this study could have been completed in early 1993 . To our knowledge, this advice was not followed and the study was never implemented.

The data we used were for 1989-1990. We hope that if the "user factor" was the cause of the elevated mortality that our study might have already led to improvements in practices. It appears that the FDA notice had that intent. 
To those readers who have heard so much rumor and innuendo about the authors that they suspect that the authors must have done something to deserve such litigation we offer the following analogy: "Henry, why are you here?" Ralph Waldo Emerson is supposed to have asked Thoreau, who was in jail for refusing to pay war taxes. "Waldo, why are you not here?" Thoreau replied.

The current litigation from Minntech must not and will not deter us and others from continuing to research dialysis-related issues and report scientific findings with potential public health implications. We do invite and welcome scientific questions and challenges. We clearly see a need for continued, quality research in this area to identify the reasons for the observed differences in mortality. Research findings are rarely an endpoint. Our studies provide new findings and answer some questions while raising new questions which need to be addressed in future research for the pursuit of truth. We hope that the medical community will demonstrate their defense of the scientific process through condemnation of inappropriate litigation. We hope that Minntech will take tangible action to repair the damage that their litigation has done to the scientific process and to show their commitment to preserving scientific inquiry.

\section{Note Added in Proof}

On February 15, 1994, very close to the time of writing of this editorial, Minntech filed a research protocol to obtain the data from the federal government.

\section{References}

1. ANON: Minntech files new lawsuit. Dial Transplant 22:232, 1993

2. Neumann ME: Minntech sues authors of new report on dialyzer reuse. Neph News Issues 7(5):8, 10; 1993

3. ANON: Scientists who blow the whistle on accepted medical treatments face a new enemy-They may become defendants in a lawsuit. Partners in Prevention Spring:5, 1993

4. ANON: Minntech flles lawsuit against "Renalin" Paper" authors. Contemp Dial Nephrot 14(6):13, 35; 1993

5. Held PJ, Wolfe RA, Gaylin DS, Pon FK, Levin NW, Turenne MN. Analysis of the association of dialyzer reuse practices and patient outcomes. Am J Kidney Dis (In press)

6. Kjellstrand CM: The search for scientifie truth versus corporate interests. Semin Dial 6:281-283, 1993

7. DePalma J: Law and truth, or all choices involve compromise. Neph News Issues 7:38-39, 1993

8. Desai S: The pitfalls of sterilants in the dialysis unit. Neph News Issues 7(11): 12, 31, 34-35; 1993

9. Ward RA: Reaction. Neph News /ssues 7:34-35, 1993

10. Miller CW: Reaction. Neph News Issues 7:34, 36; 1993

11. Tolkars JI, Alter MJ, Favero MS, Moyer LA, Bland LA: National surveillance of dialysis-associated diseases in the United States. ASAIO J 39:966-975, 1991

12. Flaherty JP, Garcia-Houchins S, Chudy R, Amow PM: An outbreak of gram-nepative bacteremia traced to contaniated $O$-rings in reprocessed dialyzers. Ann Intern Med 119:1072-1078, 1993

13. Bolan G, Reingold AL. Carson LA, Silcox VA, Woodley CL. Hayes PS, Hightower AW, McFartand L. Brown JW III, Petersen NJ, Favero MS, Good RC. Broome CV: Infections with Mycobacterium chelonei in patients receiving dialysis and using reprocessed hemodialyzers. $J$ Infect Dis 152:1013-1019, 1985

14. Lowry PW, Beck-Sague CM, Bland LA, Aguero SM, Arduino MJ, Minuth AN, Murray RA, Swenson JM, Jarvis WR: Mycobacterium chelonei infection amons patients receiving high-flux dialysis in a hemodialysis clinic in Califomia. I Infect Dis 161:85-90, 1990

15. United States General Accounting Office: Hospital Sterilants: Insufficient FDA Regulation May Pose a Public Health Risk. GAO-HRD. 93-79. Washington DC. 1993

16. Held PJ, Pauly MV, Diamond LH: Survival analysis of patients undergoing dialysis. JAMA 257:645 -650, 1987

17. Association for the Advancement of Medical instrumentation: AAMI Recommended Practice. Reuse of Hemodialyzers (AAMI ROH1986): Artington, VA, 1993 (Nathan Levin was co-chairman of the committee that produced this report)

18. Levin NW: Dialyzer reuse: A currently acceptable practice. Semin Dial 6:89-90, 1993 